\title{
Diffuse pleural thickening in asbestos workers: disability and lung function abnormalities
}

\author{
CR McGAVIN, G SHEERS \\ From the Plymouth Chest Clinic, Plymouth
}

ABSTRACT Data from 37 asbestos workers with diffuse pleural fibrosis have been analysed. None had radiological evidence of asbestosis or physiological evidence of airflow obstruction. Forty per cent had breathlessness of MRC grade 3 or higher. Vital capacity was significantly lower in the subjects in the higher grades of breathlessness and in those with greater radiographic pleural abnormality. No relationship was demonstrated between dust exposure and either radiographic abnormality or grade of breathlessness. Diffuse pleural thickening, particularly when extensive and bilateral, causes functional impairment and disability.

Benign pleural complications of exposure to asbestos fibre include hyaline and calcified plaques, acute pleuritic reactions, and diffuse pleural fibrosis. Differentiation of these abnormalities from each other and from parenchymal fibrosis is largely dependent on radiology. The pattern of calcification in plaques is characteristic and both hyaline and calcified plaques can usually be discriminated well. ${ }^{1}$ Diffuse pleural fibrosis, particularly when unilateral, is less specifically related to asbestos exposure but commonly occurs in association with plaques and parenchymal fibrosis. Radiological features of diffuse pleural fibrosis observed in the Devonport Dockyard population include obliteration of the costophrenic angle, tenting and distortion of the diaphragmatic and pericardial pleura, coarse linear shadows, "tidemarks," and "ground glass" loss of transradiancy.

Asbestos related parenchymal fibrosis is a well recognised cause of disability for which compensation can be claimed. Plaques have little effect on lung function and seldom cause disability, whereas diffuse pleural fibrosis has been associated with significant impairment of function. ${ }^{2}$ Britton $^{3}$ published data on 88 asbestos workers attending chest clinics or referred for compensation. These men had a mixture of pleural and parenchymal disease and some also had airflow obstruction, but he concluded that diffuse pleural fibrosis, particularly when severe, could contribute to disability. Diffuse pleural

Address for reprint requests: Dr CR McGavin, Plymouth Chest Clinic, Beaumont House, Plymouth PL4 9BQ.

Accepted 20 March 1984 fibrosis has not been prescribed as an industrial injury in Britain but recommendations that it should be were made by the Industrial Injuries Advisory Council in 1973 and $1982 .{ }^{4}$ This retrospective study provides further evidence on this question by examining breathlessness and lung function abnormality in a group of men with diffuse pleural fibrosis but without significant parenchymal fibrosis or airflow obstruction.

\section{Methods}

Records of workers who had been exposed to asbestos in HM Dockyard, Devonport, and who had diffuse pleural thickening were examined. Most cases had been identified in Dockyard medical surveys, but a few came from referrals to the Plymouth Chest Clinic. Subjects were included only if a chest radiograph, a questionnaire on respiratory symptoms, $\frac{D}{O}$ and results of lung function tests were available from the same period.

Breathlessness was assessed by the Medical Research Council (MRC) questionnaire on 0 respiratory symptoms; grade I indicates no breath- $\omega$ lessness on climbing hills or stairs and grade $\mathrm{V}$ indicates breathlessness at rest or on trivial exertion. $\bullet$ Lung function tests included $\mathrm{FEV}_{1}$ and forced vital capacity (FVC) measured by spirometer, total lung ? capacity (TLC), and residual volume (RV) measured by helium dilution, and carbon monoxide $\stackrel{\vec{D}}{\mathrm{D}}$ transfer factor (TLCO) and transfer coefficient $\stackrel{\rho}{\stackrel{Q}{\rho}}$

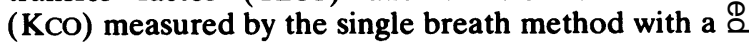
10 second breath holding time. In $64 \%$ of men the haemoglobin concentration was known and TLCO 
was corrected ${ }^{5}$ to a haemoglobin value of $14.6 \mathrm{~g} / \mathrm{dl}$. All lung function results were expressed as percentages of predicted values for age and height. ${ }^{5}$

The chest radiographs had previously been read by three experienced readers according to the ILO U/C (1971) classification, ${ }^{6}$ and all the films were read again by the authors without knowledge of the physiological or subjective assessments. The films were read on the basis of the ILO 1980 classification ${ }^{7}$ with particular attention to the profusion of small irregular opacities and to the nature and extent of pleural abnormalities. Only good quality posteroanterior radiographs were used. The revised classification allows both sides of the chest to be scored for (1) presence of plaques, diffuse thickening, or both; (2) the width of the pleural thickening in profile; ( 3 ) the presence of "face on" pleural disease; (4) the extent of the abnormality in relation to the height of the chest wall; (5) disease affecting the diaphragm; and (6) obliteration of the costophrenic angle. In practice, plaques made only a small contribution to the pleural abnormality in this series, and because they are thought not to cause disability ${ }^{2}$ they were ignored for the purpose of this study. Preliminary examination suggested that the radiological features contributing most to disability were obliteration of the costophrenic angle and the extent of the pleural thickening on the chest wall; the extent was scored as 1 if the thickening extended for less than $25 \%$ of the total height of the lung, 2 if it was 25$50 \%$, and 3 if it was greater than $50 \%$. A radiographic score has been devised based on the presence or absence of costophrenic angle obliteration (scored 1 or 0 ) plus the extent score, giving a maximum of 4 for each side of the chest and of 8 for each film. Scores above 4 imply bilateral disease but some men with scores below 4 also had bilateral disease of lesser severity.

An asbestos dust exposure rating was calculated for those men whose employment history was known in detail. Jobs were coded in groups according to an estimated degree of exposure assessed by experienced investigators in the dockyard. The job code was multiplied by the number of years spent in that job to give an exposure rating (CE Rossiter $e t$ al, paper presented to Bochum Conference on Pneumoconioses, 1983).

To make an accurate assessment of disability due to diffuse pleural fibrosis, men with significant airflow obstruction or with parenchymal fibrosis were excluded. Airflow obstruction was defined as an $\mathrm{FEV}, \mathrm{FVC}$ ratio of more than $5 \%$ below the predicted value or an RV/TLC ratio greater than $38 \%$. Parenchymal fibrosis was defined as a profusion of small irregular opacities of $1 / 1$ or more on the posteroanterior radiograph.

\section{NO OF WORKERS}

$15-$

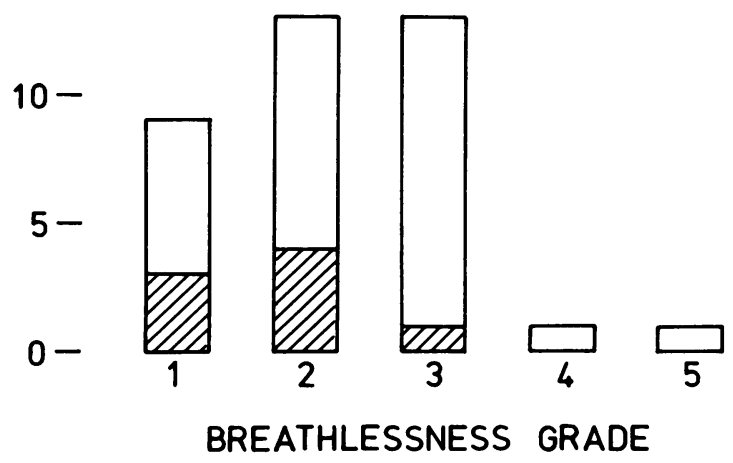

Grades of breathlessness in the 37 workers. men with unilateral disease.

\section{Results}

Thirty seven men were identified who fulfilled the criteria for diffuse pleural fibrosis without asbestos related parenchymal fibrosis or airflow obstruction. Their mean (SD) age was 54 (9) years. The distribution according to breathlessness grade is shown in the figure. Fifteen $(40 \%)$ were in grades 3,4 , and 5-that is, they claimed to be unable to walk on level ground at a normal pace without breathlessness. Eight men had unilateral pleural disease (fig). They were predominantly in breathlessness grades 1 and 2; their mean grade was 1.75 , compared with 2.35 in the 29 men with bilateral disease.

Table 1 shows mean lung function values (expressed as percentage of predicted values) according to grade of breathlessness. Grades 1 and 2 have been combined and grades 3, 4, and 5 have been combined to give two groups because of the small numbers. The mean values of FEV, FVC, and TLC for both groups fell below $90 \%$ of the predicted values and the mean RV for both groups fell below $80 \%$ of the predicted values. Mean FEV 1 and FVC were significantly lower in the higher breathlessness grades and the difference in TLC was of borderline significance ( $p=0.052$, Student's $t$ test). Exclusion of the eight men with unilateral disease did not affect the mean lung function values in the groups but, because of the smaller size of the sample, statistical significance was no longer reached.

Breathlessness is compared with radiographic score in table 2. The breathlessness grades are again subdivided into two groups as in table 1 , and the radiographic scores have been divided into three 
Table 1 Results of lung function tests and breathlessness grades

\begin{tabular}{|c|c|c|c|c|c|c|c|}
\hline \multirow[t]{2}{*}{ Breathlessness grade } & \multirow[t]{2}{*}{$n$} & \multicolumn{6}{|c|}{ Lung function values (mean \% predicted) } \\
\hline & & $F E V_{1}$ & FVC & $R V$ & $T L C$ & TLCo & Kсо \\
\hline $\begin{array}{l}1,2 \\
3,4,5 \\
p\end{array}$ & $\begin{array}{l}22 \\
15\end{array}$ & $\begin{array}{l}88 \\
77 \\
<0.05\end{array}$ & $\begin{array}{l}87 \\
78 \\
<0.05\end{array}$ & $\begin{array}{l}77 \\
73 \\
\text { NS }\end{array}$ & $\begin{array}{l}82 \\
74 \\
\text { NS }\end{array}$ & $\begin{array}{l}89 \\
84 \\
\text { NS }\end{array}$ & $\begin{array}{l}106 \\
111 \\
\text { NS }\end{array}$ \\
\hline
\end{tabular}

FVC—forced vital capacity; RV—residual volume; TLC—-total lung capacity; TLco—transfer factor; Kco—transfer coefficient; NS—not significant.

Table 2 Radiographic scores and breathlessness grades

\begin{tabular}{lcccc}
\hline $\begin{array}{l}\text { Breathlessness } \\
\text { grade }\end{array}$ & \multicolumn{4}{l}{ Radiographic score } \\
\cline { 2 - 5 } & & $1-4$ & $5-6$ & $7-8$ \\
\hline 1,2 & 22 & 11 & 9 & 2 \\
$3,4,5$ & 15 & 4 & 3 & 8 \\
\hline
\end{tabular}

The results show a significant association: $p<0.025\left(\chi^{2}\right.$ test $)$.

groups. A significant relationship was found, higher radiographic scores being associated with increasing breathlessness ( $\chi^{2}$ test). Exclusion of the eight men with unilateral disease reduced the size of the sample to the extent that the $\chi^{2}$ test could not be applied with confidence.

The relationship between radiographic score and lung function (table 3 ) was examined by one way analysis of variance. There is a highly significant decline in $\mathrm{FEV}_{1}$ and FVC with increasing radiographic score. An important reduction of volume was seen in the men with the highest radiographic scores ( 7 and 8 )-that is, those with extensive bilateral pleural disease. There were no significant differences in RV and TLCO, although the differences in TLC approached significance $(p=0.06)$. The significant reduction of $\mathrm{FEV}_{1}$ and $\mathrm{FVC}$ in the group with the highest radiographic scores was unaffected by the exclusion of the eight men with unilateral disease.

KCO rose from a mean of $92 \%$ in those with radiographic scores $1-3$ to $114 \%$ for scores $4-6$, and to $112 \%$ for scores 7 and 8 . This increase is significant $(p<0.025)$.

The dust exposure rating was known for 33 of the 37 men. There was no relationship between dust exposure and either radiographic score $(r=0.08)$ or breathlessness grade $(r=0.05)$.

\section{Discussion}

There are inevitable methodological problems inherent in this study. Firstly, it is a retrospective analysis of data collected mainly in dockyard surveys with no objective assessment of disability. Secondly, it is not certain that in all cases the pleural thickening was due to asbestos. This is particularly relevant $\overrightarrow{0}$ in the eight men with unilateral disease. It is well $\stackrel{\infty}{\oplus}$ recognised that diffuse pleural fibrosis may develop in a stepwise rather than slowly progressive fashion, ${ }^{3}$ and we have seen unilateral disease occurring as a stage in the early development of bilateral diffuse pleural fibrosis in dockyard workers under observation. Furthermore, there is no reason to suppose that the physiological effects of diffuse pleural fibrosis from asbestos exposure are any different from those associated with other diseases causing pleural fibrosis. A third problem is the possible contribution of pulmonary fibrosis to disability and to lung function abnormality. It is known that a degree of asbestos related parenchymal fibrosis can be present without radiological abnormality. ${ }^{8}$ The presence of pleural fibrosis makes radiological assessment of the underlying lung more difficult. We have attempted to avoid including patients with appreciable lung disease by excluding any with a profusion score of $1 / 1$ or more. Two features of the results suggest that lung disease has not played an important role in causing disability. The first is that KCO is of seen to rise with increasing radiographic score-a $N$ feature of pleural rather than pulmonary disease. 9

Table 3 Radiographic scores and results of lung function tests

\begin{tabular}{llllllll}
\hline Radiographic score & $n$ & \multicolumn{1}{l}{ Lung } & function values (mean \% predicted) & & \\
\cline { 2 - 7 } & & FEV & FVC & TLC & RV & TLco \\
\hline $1-4$ & 15 & 90 & 88 & 83 & 76 & 86 \\
$5-6$ & 12 & 87 & 87 & 79 & 79 & 92 \\
$7-8$ & 10 & 72 & 71 & 72 & NS & NS \\
p & & $<0.005$ & $<0.005$ & NS & & \\
\hline
\end{tabular}

Abbreviations as in table 1 . 
The second is that there is no evidence of any relationship between dust exposure and breathlessness in this study, a relationship which might be expected to be present if asbestos related parenchymal fibrosis was contributing to disability.

The lack of correlation between exposure ratings and disability raises queries. Pleural reactions as a whole are related to exposure but it is possible that the evolution of diffuse pleural fibrosis in particular may not be dose related. It is evident that there is wide individual variation in the extent of the fibrosis which ultimately results from these pleural reactions.

Although the survey identified some men with mild and moderate disability, there were few with severe disability (breathlessness grades 4 and 5). This could be because even extensive pleural thickening does not necessarily cause severe disability, but another possibility is that very disabled men had left the dockyard, and thus avoided detection by surveys. Alternatively, men with extensive bilateral disease may have been excluded from the survey because the readers of the radiographs could not satisfy themselves about the absence of small irregular pulmonary opacities.

Despite these difficulties we believe that our group consists of asbestos workers with a spectrum of respiratory disability arising from diffuse pleural fibrosis rather than from asbestos related parenchymal fibrosis or airflow obstruction. Furthermore, the method of selection of this sample will have led to a more accurate assessment of disability than would be likely to result from the investigation of men applying for compensation.

The results show that $40 \%$ of this group of men with diffuse pleural fibrosis were disabled to the extent that they were unable to walk along level ground at a normal pace. Vital capacity was significantly inversely correlated both with increasing breathlessness grade and with increasing radiographic pleural abnormality. The data suggest that men with lower radiographic scores, inevitably including all those with unilateral pleural disease, are less disabled than those with higher scores. The results support the hypothesis that diffuse pleural fibrosis is a cause of impaired lung function and of disability, irrespective of the presence of other asbestos related disease.

We are grateful to Surgeon Commander GHG McMillan, Royal Navy, for allowing access to data collected in the medical research unit of HM Dockyard, Devonport, and we gratefully acknowledge help given by Mrs Ruth Coles in the collection of data. We also thank Mrs Julie Hewitt for expert secretarial assistance and Dr S Shaw, Plymouth Polytechnic, for statistical analysis and advice.

\section{References}

${ }^{1}$ Fletcher DE, Edge JR. The early radiological changes in pulmonary and pleural asbestosis. Clin Radiol 1970;21:355-65.

${ }^{2}$ Lumley KPS. Physiological changes in asbestos pleural disease. In: Walton WH, ed. Inhaled particles $I V$. Oxford: Pergamon Press, 1977:781.

${ }^{3}$ Britton MG. Asbestos pleural disease. Br J Dis Chest 1982;76:1-10.

4 Industrial Injuries Advisory Council. Asbestosis-related diseases without asbestosis. London: HMSO, 1982.

${ }^{5}$ Cotes JE. Lung function: assessment and application in medicine. 3rd ed. Oxford: Blackwell Scientific Publications, 1975:281-7.

' ILO U/C. International classification of radiographs of pneumoconioses. Geneva: International Labour Office, 1971. (Occupational Safety and Health Series No 22.)

${ }^{7}$ ILO. International classification of radiographs of pneumoconioses. Geneva: International Labour Office, 1980. (Occupational Safety and Health Series, No 22.)

${ }^{8}$ Harries PG. RN Clinical Research Working Party 1971: Report No 1/71:114. Gosport, Hants: Institute of Naval Medicine, 1971.

9 Ogilvie CM, Forster RE. Single breath transfer factor 25 years on: a reappraisal. Thorax 1983;38:1-9. 